\title{
Retraction Note to: Effect of RAB31 silencing on osteosarcoma cell proliferation and migration through the Hedgehog signaling pathway
}

\author{
Published online: 3 February 2022 \\ (c) The Japanese Society Bone and Mineral Research 2022 \\ Retraction Note to: \\ Journal of Bone and Mineral Metabolism \\ (2019) 37:594-606 \\ https://doi.org/10.1007/s00774-018-0961-9
}

Qiong $\mathrm{Yu}^{1} \cdot$ Dong $\mathrm{Li}^{2} \cdot$ Dan Wang ${ }^{3} \cdot$ Chun-Mei Hu${ }^{1} \cdot$ Yan Sun ${ }^{1} \cdot$ Yan Tang ${ }^{1} \cdot$ Guang Shi ${ }^{1}$

The authors have retracted this article. After publication the authors discovered that they were unable to repeat their results. In this article the authors concluded that silencing the rab31 gene inhibits hedgehog pathway activation, but in the repeated experiments the authors found that the Hedgehog pathway was not activated when the rab31 gene was over-expressed. All authors agree to this retraction.

Publisher's Note Springer Nature remains neutral with regard to jurisdictional claims in published maps and institutional affiliations.

The original article can be found online at https://doi.org/10.1007/ s00774-018-0961-9.

Guang Shi

shiguang_sg11@163.com

1 Department of Hematology and Oncology, The Second Hospital of Jilin University, No. 218, Ziqiang Street, Changchun 130041, Jilin, People's Republic of China

2 Department of Obstetrics and Gynecology, The Second Hospital of Jilin University, Changchun 130041,

People's Republic of China

3 Department of Breast Surgery, The Second Hospital of Jilin University, Changchun 130041, People's Republic of China 